The CASE: A 5-year-old black girl, who was originally from Sudan, was seen by her general pediatrician after her parents noted intermittent bright red blood staining her underwear. She did not report abdominal pain, constipation, dysuria, incontinence or fever. She was not taking any medications and had no known allergies. Her past medical history was significant for a partial circumcision performed for cultural reasons in her country of origin. A physical examination revealed evidence of type I female circumcision, with partial removal of the clitoris and a doughnut-shaped mass with bright red blood in the introitus. A pelvic ultrasound was normal, and she then underwent examination under anesthesia by a pediatric gynecologist. Fig. 1 shows an intact hymen (partially obscured by the urinary catheter), without signs of trauma or of a foreign body. A Foley catheter placed in the doughnut-shaped mass through the urethra drained urine, which suggested urethral prolapse. Cystoscopy confirmed the diagnosis.

The prolapse was temporarily reduced by simple manual manipulation. The family declined topical estrogen therapy and surgical intervention but agreed to a regimen of sitz baths (sitting in clear, lukewarm water for 20 minutes, 4 times a day). The prolapse recurred a few weeks later but resolved again with manual manipulation in the pediatrician's office. Three months later, the child presented with vaginal pruritis, dysuria and another recurrence of the prolapse. Vaginal swabs for bacteria and urine culture were negative. The prolapse was again manually resolved, and the family agreed to the application of topical estrogen twice daily for 2 weeks. Nine months later, the prolapse had not recurred.

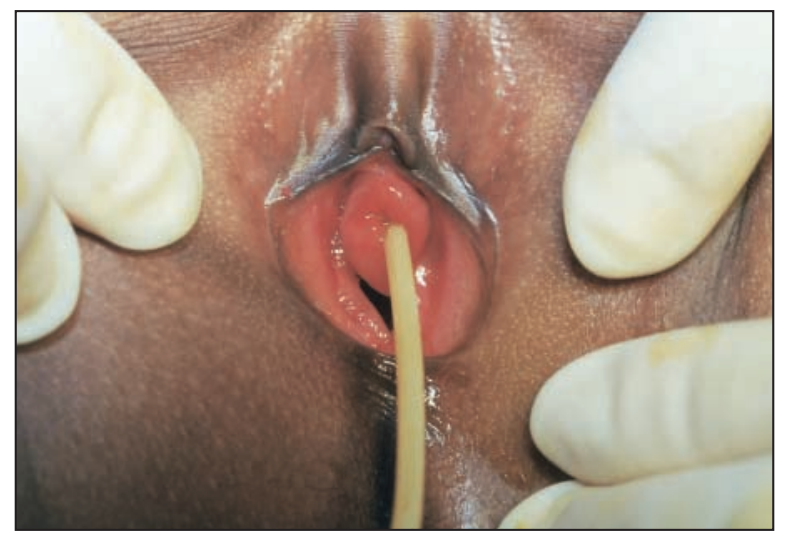

$\mathrm{U}$ rethral prolapse among girls has an estimated incidence of 1 in $2880 . .^{1}$ It is more common among prepubescent black girls, who usually present between the ages of 4 and 8 years, but it is also common among menopausal white women. ${ }^{2}$ It is unclear why it occurs more frequently among black girls, but prepuberty and menopause are associated with a relative estrogen deficiency, especially at the distal portion of the female urethra, an area with many estrogen receptors. Factors contributing to urethral prolapse include estrogen deficiency, intra-abdominal pressure (e.g., coughing, large weight for age), trauma, urinary tract infection and anatomical defects, which include female circumcision (or female genital mutilation). Box 1 shows the classification of the different types of female circumcision. ${ }^{3}$ Higher degrees, with their greater degree of anatomic compromise, may have a greater association with urethral prolapse, but this is understudied.

Urethral prolapse is often asymptomatic, but it can also present as painless vaginal bleeding. Although rare, large masses may lead to urinary retention and secondary dysuria due to bladder inflammation and infection. Classification is based on the degree of prolapse and inflammation (Box 2). Grade II, which was seen in our patient, is

Box 1: Classification of female circumcision (or female genital mutilation) ${ }^{4}$

Type I: Removal of skin surrounding the clitoris, with the clitoris left intact, partially excised or entirely excised

Type II: Removal of the entire clitoris and all or part of the labia minora

Type III: Partially covering the urethra and introitus by stitching together raw surfaces created in the labia majora

Type IV: Clitoral, labial and vaginal damage using a variety of strategies (stretching, piercing, pricking, cauterizing, corrosive substances)

Box 2: Classification of urethral prolapse $^{3}$

Grade I: Minimal or segmental prolapse without inflammation Grade II: Circumferential prolapse with edema

Grade III: Edematous mass protruding beyond the labia minora

Grade IV: Severe hemorrhagic inflammation or necrosis or ulceration of the prolapse 
Practice

the most common, followed closely by grade III. ${ }^{2}$ As shown in Fig. 1, the mass usually encircles the urethral meatus.

The differential diagnosis of urethral prolapse includes a variety of benign, malignant, structural, infectious and traumatic causes (Box 3). To help distinguish between a urethral

Box 3: Causes of vaginal bleeding

\section{Mass present}

- Structural: prolapse of urethra, cervix or bladder; ureterocele; ectopic ureter; hydrometrocolpos

- Infectious: periurethral abscess

- Malignant: sarcoma botryoides

- Benign: polyp, condyloma acuminate, hemangioma

- Traumatic: postsurgical, straddle injury, sexual assault, foreign body

No mass

- Infectious: vulvovaginitis

- Traumatic: postsurgical, straddle injury, sexual assault, foreign body

- Vascular: menarche or menstruation, coagulopathy and vaginal mass, the vulva can be retracted downward and laterally; likewise, the knee-chest position with the child supine can help visualize the anatomy. Examination under anesthesia may be required if the diagnosis remains uncertain.

Not enough is known about the natural history of urethral prolapse to know how often it resolves spontaneously. Nonsurgical management options include sitz baths (which are thought to reduce the inflammation), oral and topical antibiotics (to control possible infection) and topical estrogen cream. Current evidence supports the use of $0.5 \%$ estrogen cream twice daily for 4-6 weeks in combination with sitz baths as first-line therapy; however, this may be successful in only one-third of cases. ${ }^{2}$ Surgery can offer definitive treatment, ${ }^{2,4}$ but it may not be the first choice for some families for a number of reasons, including cultural ones. Surgical excision should be re- served for those prepubertal patients for whom conservative therapy does not work, patients with severe (grade IV) prolapse and postpubertal patients. ${ }^{2}$

\section{Mia E. Lang}

Child Health Ambulatory Clinic

Azza Darwish

Royal Alexandra Hospital

Ann Marie Long

Hys Medical Centre

Edmonton, Alta.

Acknowledgements: We wish to thank the members of the media department at the Royal Alexandra Hospital for their assistance with the photograph.

\section{References}

1. Owens SB, Morse WH. Prolapse of the female urethra in children. 7 Urol 1968;100:171-4.

2. Venugopal S, Duncan ND, Carpenter R. Urethral prolapse in girls; a series of 27 cases. Pediatr Surg Int 1995;10:115-7.

3. American Academy of Pediatrics Policy Statement. Female genital mutilation. Pediatrics 1998;102:153-6.

4. Valerie E, Gilchrist BF, Frischer J, Scriven R, Klotz DH, Ramenofsky ML. Diagnosis and treatment of urethral prolapse in children. Urology 1999:54:1082-4.

\section{THE FRAMEWORK}

\section{for HIGH STANDARDS ard DEDICATION}

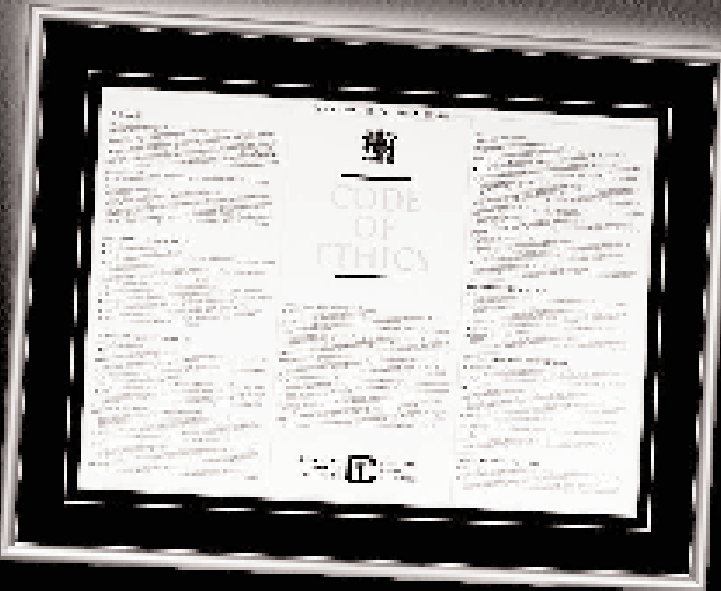

Patients rebr an you for your expertive, ompresionand integriby.

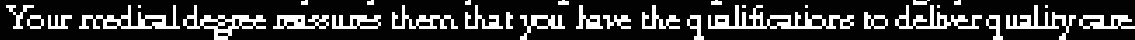

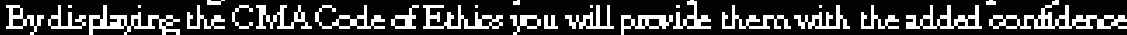
that you hove the hish sandand and dedization to always wodk in their test interst.

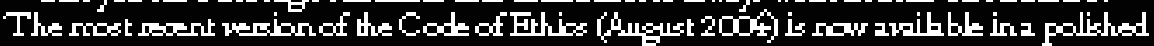

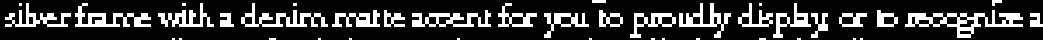
ollogesue for their onmitrent and redial profesibraliem
CAHAD LAH MEDLCAL ASOOCLATLOH

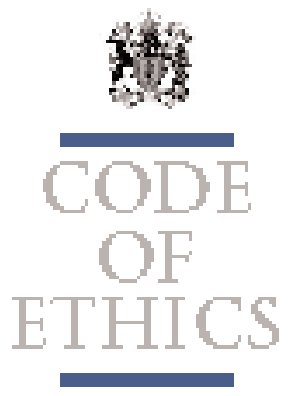

Woung be pour oodec by tele phore throust the Clive Member Service Centue at $1888855-2555$

$o$ ronline 가 crna.cal crmaboolsthtm Online odecinsis zuible to vejered uess of $\mathrm{cm}$ an vecistan ion fire to Curadian p tysiciens and orhy als: 2 minuk.

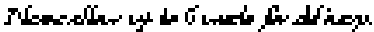

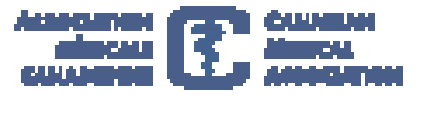

